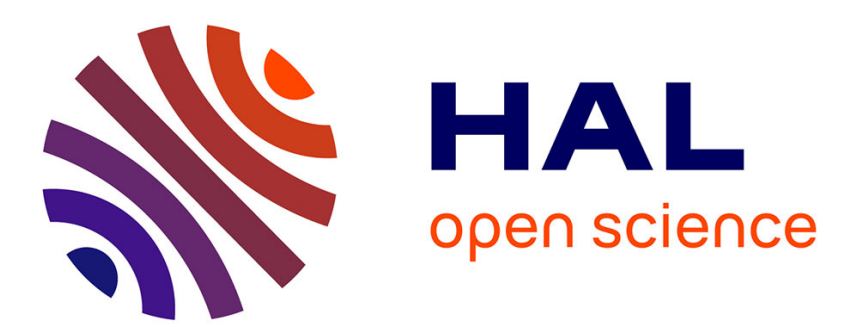

\title{
Caractérisation de la stabilité d'un élément piézoélectrique du type PZT sous compression uniaxiale rapide
}

\author{
H. Ohanessian, P. Gonnard, M. Troccaz, L. Eyraud, P. Eyraud
}

\section{- To cite this version:}

H. Ohanessian, P. Gonnard, M. Troccaz, L. Eyraud, P. Eyraud. Caractérisation de la stabilité d'un élément piézoélectrique du type PZT sous compression uniaxiale rapide. Revue de Physique Appliquée, 1983, 18 (8), pp.479-486. 10.1051/rphysap:01983001808047900 . jpa-00245109

\section{HAL Id: jpa-00245109 https://hal.science/jpa-00245109}

Submitted on 1 Jan 1983

HAL is a multi-disciplinary open access archive for the deposit and dissemination of scientific research documents, whether they are published or not. The documents may come from teaching and research institutions in France or abroad, or from public or private research centers.
L'archive ouverte pluridisciplinaire HAL, est destinée au dépôt et à la diffusion de documents scientifiques de niveau recherche, publiés ou non, émanant des établissements d'enseignement et de recherche français ou étrangers, des laboratoires publics ou privés. 


\title{
Caractérisation de la stabilité d'un élément piézoélectrique du type PZT sous compression uniaxiale rapide
}

\author{
H. Ohanessian, P. Gonnard, M. Troccaz, L. Eyraud et P. Eyraud \\ Laboratoire de Génie Electrique et Ferroélectricité, Institut National des Sciences Appliquées, \\ 20, avenue Albert-Einstein, 69621 Villeurbanne, France
}

(Reçu le 13 avril 1983, accepté le 13 mai 1983)

\begin{abstract}
Résumé. - Le présent article concerne la caractérisation de céramiques piézoélectriques au zirconotitanate de plomb destinées à l'allumage réversible par impact. Les mesures d'énergie complétées par des tests de stabilité sous 400 impacts de durée différente font apparaître les paramètres essentiels suivants : la stabilité est une fonction décroissante de cette durée; la qualité du matériau dépend de l'état lacunaire et de l'équilibre électrique de sa composition ainsi que du rapport des concentrations en zirconium et en titane.
\end{abstract}

\begin{abstract}
The purpose of this paper is the characterization of lead zirconate titanate piezoelectric ceramics suitable for reversible impact-striking applications. Energy-measurements and stability tests under various pulsewidth impacts were achieved; it is found that the main parameters are the following : the stability is a decreasingfunction of this pulse-width; both efficiency and stability of material depends on the vacancy-state and electric balance of this composition, as also on the $\mathrm{Zr} / \mathrm{Ti}$ ratio.
\end{abstract}

\section{Introduction.}

On se propose de définir des tests spécifiques permettant la caractérisation des céramiques piézoélectriques destinées à l'allumage réversible par impact.

Ces tests doivent mettre en évidence deux propriétés essentielles du matériau : la stabilité des caractéristiques piézoélectriques sous des contraintes répétitives et les qualités énergétiques intrinsèques de la céramique. A l'aide de ces tests, il est possible de déterminer les composés stœchiométriques ou faiblement lacunaires les mieux adaptés à l'allumage par impact.

\section{Techniques de caractérisation.}

1.1 Dispositifs expérimentaux. - On dispose de trois montages expérimentaux de compression par impact et d'une presse pour les essais en compression quasi statique.

1.1.1 Système percuteur à briquet. - Le dispositif de la figure 1 s'inspire de celui des allumeurs industriels. Un ressort taré, à l'extrémité duquel est placé le percuteur, est comprimé par l'intermédiaire d'une came. Un moteur à vitesse variable permet de faire varier la rotation de la came de 1 à 10 tours par minute. En regard de cet ensemble se trouve placé l'échantillon cylindrique de $16 \mathrm{~mm}$ de longueur et de $6,35 \mathrm{~mm}$ de diamètre coulissant sans jeu dans un support isolant en afcodur. Une enclume en acier trempé à tête semisphérique surmonte l'échantillon. Après la percussion en fin de révolution de la came, un ressort de rappel ramène le percuteur en position initiale.

Les forces appliquées, réglées par le ressort taré et la distance percuteur-enclume, sont définies par rapport au comportement à vide d'une céramique de référence présentant un coefficient $g_{33} \simeq 20 \times$ $10^{-3} \mathrm{Vm} / \mathrm{N}$. Le champ électrique est limité à environ $1,25 \times 10^{6} \mathrm{~V} / \mathrm{m}$ correspondant à une tension à vide de $20 \mathrm{kV}$ pour une céramique de $16 \mathrm{~mm}$ d'épaisseur.

La pression maximum est dans ce cas de 600 bars environ. Quant à la durée de l'impulsion de pression, elle dépend des masses percutantes mises en jeu; elle a été réglée ici à $20 \mu$ s pour l'impulsion principale (Fig. 2a). Les nombreux rebonds de faible amplitude ont peu d'influence sur les résultats.

Les essais ont été limités à 400 percussions avec une fréquence de répétition de un coup toutes les $6 \mathrm{~s}$.

1.1.2 Système percuteur à bille. - La bille de masse $m$ est lâchée par un électro-aimant depuis une hauteur $h$ au-dessus de l'enclume (Fig. 3). La céramique piézoélectrique est insérée dans un ensemble identique à celui du percuteur à briquet. On a réglé la hauteur $h$ afin d'obtenir une amplitude de $20 \mathrm{kV}$ comme précé- 


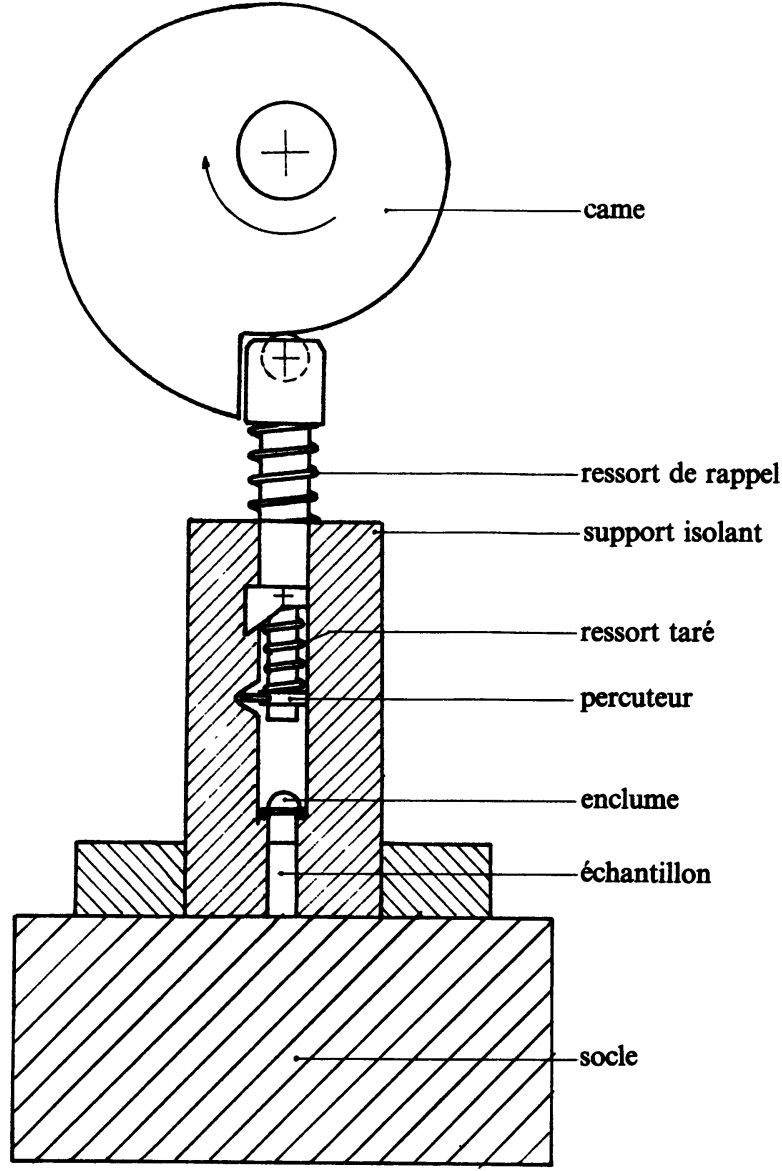

Fig. 1. - Percuteur à briquet.

[Impact-Striking type igniter.]

demment. Par contre la nature, la forme et les dimensions de l'enclume ont été étudiées de manière que le choc ait une forme sinusoïdale [2]. Ceci a conduit à une impulsion principale de $85 \mu$ s à $100 \mu$ s de largeur

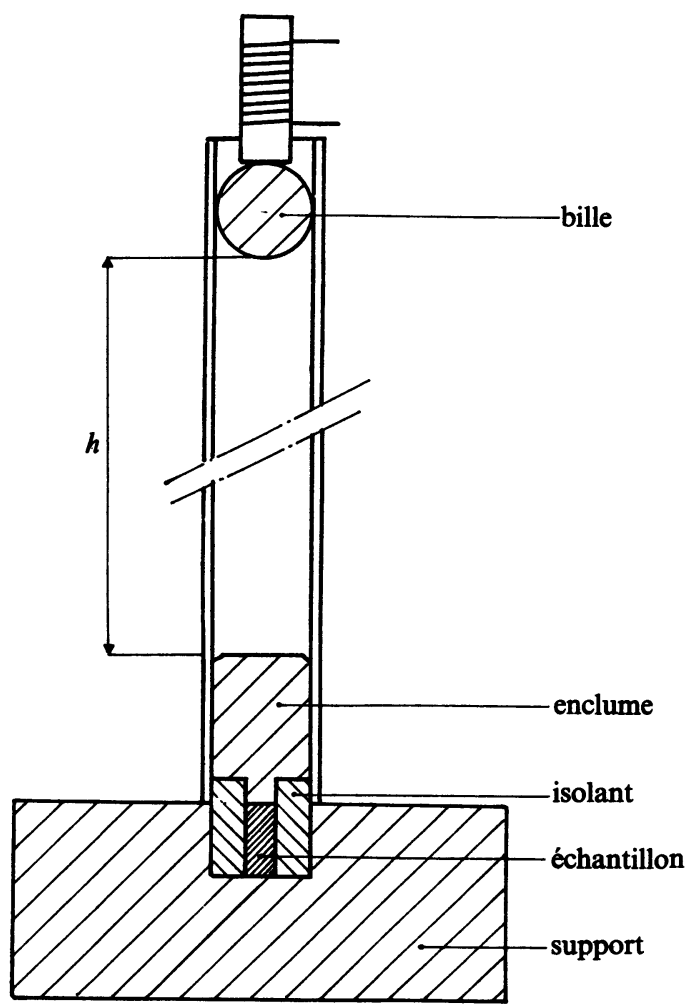

Fig. 3. - Percuteur à bille.

[Falling-Ball striking.]

avec des oscillations quasi inexistantes (Fig. 2b). La bille rebondit sur l'échantillon pour retomber ensuite mais le délai entre le premier et le deuxième choc est grand devant la durée de $100 \mu$ s et n'est donc pas pris en compte dans le résultat final. Comme pour la manipulation précédente, on procède à environ 400 impacts à une fréquence de $0,2 \mathrm{~Hz}$ environ.

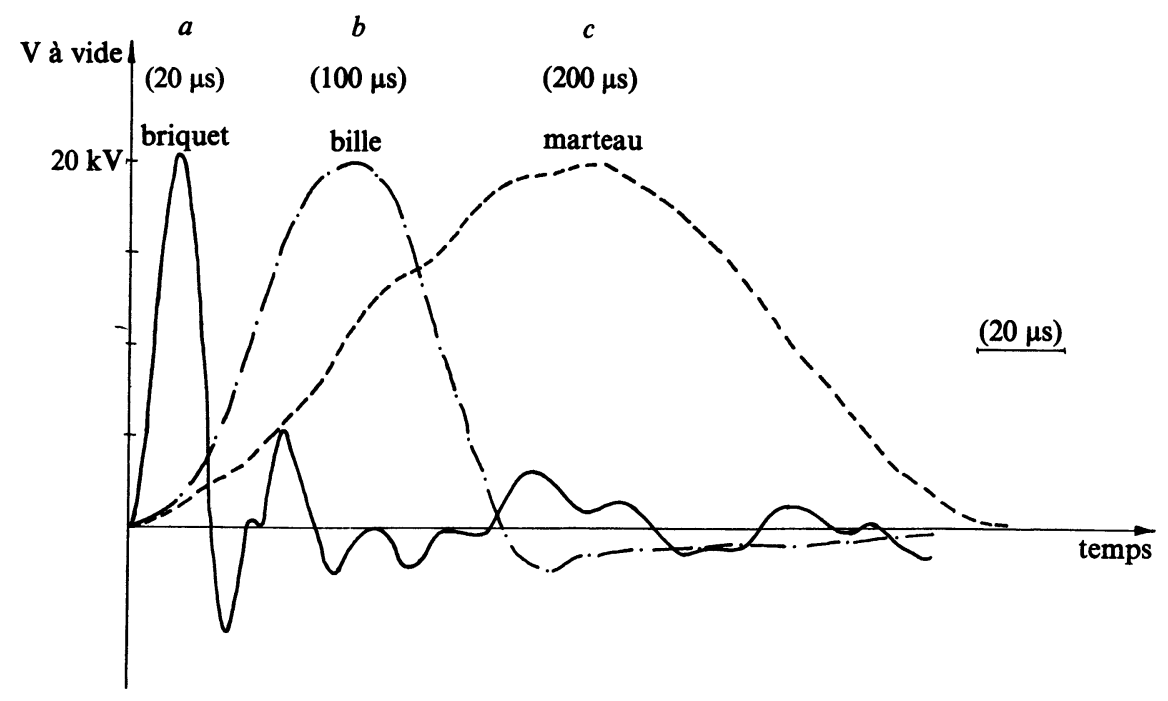

Fig. 2. - Tensions à vide, images de l'impulsion de pression.

[Open-circuit voltage versus time (pressure pulse).] 


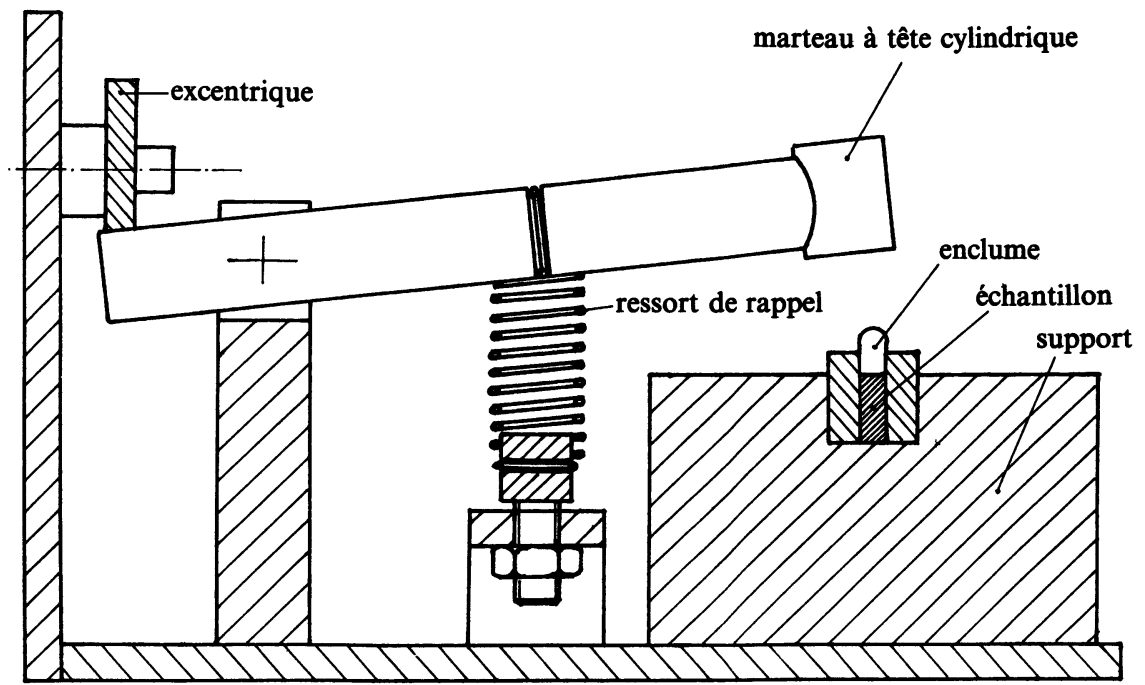

Fig. 4. - Percuteur à marteau.

[Hammer striking.]

1.1.3 Système percuteur à marteau. - Le troisième montage (Fig. 4) consiste en un marteau dont la tête cylindrique en acier trempé a une surface de $360 \mathrm{~mm}^{2}$ et une hauteur de $24 \mathrm{~mm}$. Elle est portée par un bras mû verticalement par un moteur par l'intermédiaire d'un galet excentré. Un ressort de rappel dont la force est tarée par un système réglable assure la répétitivité du choc; la tension à vide est encore réglée à environ $20 \mathrm{kV}$ sur la céramique étalon. On utilise un ensemble support échantillon identique aux précédents. Dans cette expérience, la durée du choc est nettement plus importante que les précédentes : de 180 à $200 \mu \mathrm{s}$ pour une compression maximum de 600 bars encore (Fig. 2c). Nous effectuerons 400 impacts dans les mêmes conditions de répétition que précédemment.

1.1.4 Système à compression quasi statique. - Un ensemble support-échantillon, identique aux précédents, est placé sous une presse uniaxiale (Fig. 5).

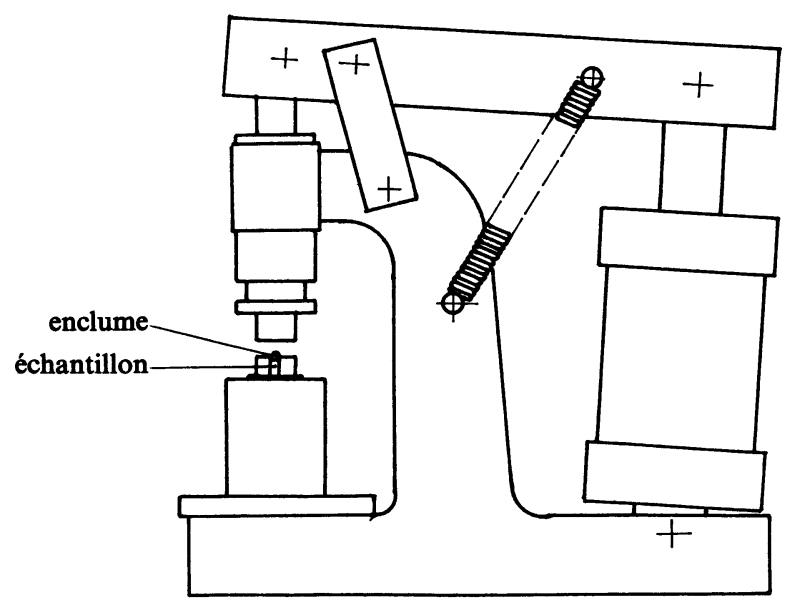

Fig. 5. - Presse uniaxiale.

[Uniaxial press.]
Des échantillons sont soumis à des compressions de 600 bars avec des temps de montée de l'ordre de 2 à $5 \mathrm{~s}$. Il est également possible avec ce montage de procéder à une dépolarisation irréversible de l'échantillon par compression à 3000 bars. Les charges écoulées en court-circuit sur un condensateur de $10 \mu \mathrm{F}$ sont mesurées à l'aide d'un amplificateur opérationnel alors que la contrainte est déterminée par une rondelle de charge à quartz suivie d'un amplificateur de charge Kistler. La courbe $Q=f(T)$ peut ainsi être tracée directement.

1.2 TeSTS EFFECTUÉs. - Chaque échantillon est caractérisé par quatre types de tests.

1.2.1 Test de mesure d'énergie. - Le procédé utilisé décrit dans un précédent article [1] consiste à transférer par l'intermédiaire d'un pont redresseur sur une capacité adéquate l'énergie électrique libérée par la céramique piézoélectrique au cours d'une compression uniaxiale impulsionnelle produite par le système percuteur à bille.

On détermine l'énergie maximum $W_{\mathrm{M}}$ transférée en faisant varier le condensateur de charge.

1.2.2 Test de stabilité sous étincelles. - Cet essai est effectué à l'aide du système percuteur à briquet; l'échantillon est relié à des électrodes à pointes distantes de $7 \mathrm{~mm}$ entre lesquelles éclate l'étincelle (Fig. 6a). La tension de claquage est de l'ordre de $7 \mathrm{kV}$. Cette première expérience permet de déterminer la stabilité des céramiques dans les conditions d'utilisation industrielle. Elle est caractérisée par la variation relative du coefficient piézoélectrique entre le début $d_{33_{0}}$ et la fin $d_{33_{\mathrm{L}}}$ de l'expérience.

$$
\frac{\Delta d}{d}=\frac{d_{33_{\mathrm{L}}}-d_{33_{0}}}{d_{33_{0}}} \quad(\% / \mathrm{oo})
$$




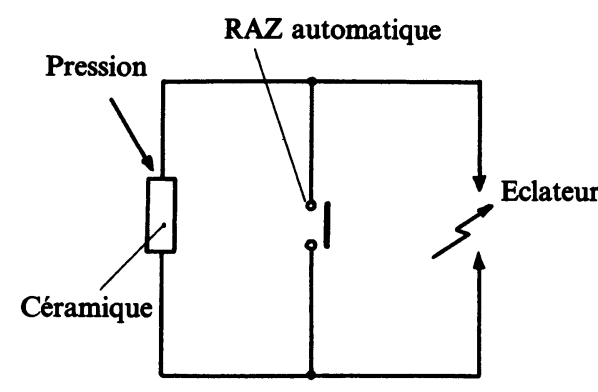

(a)

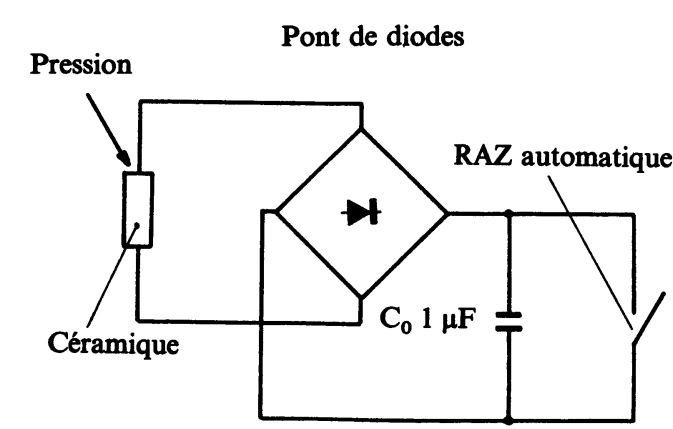

(b)

Fig. 6. - Schémas électriques de mesure.

[Measuring devices.]

1.2.3 Test de stabilité en court-circuit. - Cette série d'expériences est plus quantitative; elle donne des informations non seulement avant et après le test, mais permet également de suivre l'évolution du comportement des échantillons à chaque instant de la manipulation.

A l'aide des trois systèmes percuteurs, on effectue des mesures de même nature sur des céramiques issues du même lot. On utilisera chaque fois le montage de la figure $6 b$. La céramique est chargée par un condensateur de forte valeur vis-à-vis de sa capacité propre

$$
C_{0}=1 \mu \mathrm{F} \gg C_{\text {céramique }} \simeq 40 \mathrm{pF} .
$$

Cette valeur constitue en fait un court-circuit pour la céramique aux fréquences équivalentes des impulsions de pression (de $5 \mathrm{kHz}$ à $50 \mathrm{kHz}$ ). La liaison générateur piézoélectrique-charge non linéaire s'effectue à travers un pont de Graetz constitué par des diodes rapides BA159. Ceci permet de récupérer les charges aussi bien pendant la montée que pendant la descente de pression. On mesure ainsi la charge électrique totale produite par le choc. On effectue alors les mesures suivantes pour chaque expérience :

- mesure du coefficient piézoélectrique initial $d_{33_{0}}$,

- mesure des charges électriques totales $\Delta Q$ écoulées à chaque impulsion de pression,

- mesure du coefficient piézoélectrique final après 400 cycles $d_{33_{L}}$.

Ces différentes informations permettent d'atteindre deux critères importants de stabilité des échantillons :

\section{$\Delta d / d$,}

- la variation relative du coefficient piézoélectrique

- la variation relative des charges écoulées entre la charge maximum $Q_{\mathrm{m}}$ et la charge finale $Q_{\mathrm{L}}$

$$
\frac{\Delta Q}{Q}=\frac{Q_{\mathrm{L}}-Q_{\mathrm{m}}}{Q_{\mathrm{m}}} \quad(\%) .
$$

1.2.4 Test sous contrainte uniaxiale quasi statique. $\mathrm{La}$ variation des charges écoulées et la variation relative du coefficient piézoélectrique sont mesurées après 10 compressions puis 30 compressions à 600 bars.
On procède enfin à l'enregistrement des charges écoulées sous compression élevée, jusqu'à 3000 bars.

1.3 Analyse aUX RAYONS X. - Pour déterminer l'importance relative des phases rhomboédrique (R) et tétragonale $(\mathrm{T})$ dans les compositions étudiées, on utilise la diffraction X. En employant un diffractomètre Siemens avec une anticathode au cobalt, on enregistre les diagrammes de poudre des différents échantillons à température ambiante. En se référąnt aux travaux de Mabud [3], on a utilisé la réflexion 310 qui donne deux raies 013 et 310 dans la phase tétragonale et également deux raies 310 et $\overline{3} 10$ dans la phase rhomboédrique, ces deux dernières étant pratiquement confondues. Ces raies correspondent à des angles de diffraction $2 \theta$ compris entre $86^{\circ}$ et $90^{\circ}$ dans l'ordre croissant suivant :

$$
(013)_{\mathrm{T}} ;(310+\overline{3} 10)_{\mathrm{R}} ;(310)_{\mathrm{T}} .
$$

Pour connaître la proportion des phases (R) et (T), on calcule le rapport des surfaces de la raie $(013)_{\mathrm{T}}$ à la surface totale du groupe de raies; en effet, la raie $(013)_{\mathrm{T}}$ reste toujours discernable et isolée, alors que les raies $(310)_{\mathrm{T}}$ et $(310, \overline{3} 10)_{\mathrm{R}}$ présentent dans tous les cas un chevauchement important.

\section{Résultats expérimentaux.}

\subsection{ChOIX DES MATÉRIAUX.}

2.1.1 Classement des matériaux. - La plupart des auteurs classent les matériaux piézoélectriques pour génération d'étincelles en trois catégories [4-6].

- Les matériaux de type "doux " dont les propriétés piézoélectriques et électromécaniques sont améliorées par substitution d'ions hétérovalents donneurs tels que $\mathrm{La}^{3+}$ à la place de $\mathrm{Pb}^{2+}$ et $\mathrm{Nb}^{5+}$ ou $\mathrm{W}^{6+}$ à la place de $(\mathrm{ZrTi})^{4+}$. Ces matériaux à lacunes cationiques sont connus pour présenter une importante dégradation des performances tant dans les systèmes à compression statique ("squeeze ") que dans les systèmes dynamiques (" choc »). 
- Les matériaux de type " dur " à champ coercitif et facteur de qualité mécanique élevés. Ces compositions, obtenues par substitution d'ions hétérovalents accepteurs ( $\mathrm{Fe}, \mathrm{Cr}, \mathrm{Mn}, \mathrm{Ni}$, etc...) en remplacement de (ZrTi) sont lacunaires en oxygène. Elles présentent une grande stabilité dans les systèmes à compression mais des performances électromécaniques médiocres.

- Les matériaux intermédiaires que l'on peut qualifier de "semi-dur" ou "semi-doux " obtenus par substitutions hétérovalentes sur un site ou sur chaque site $\mathbf{A}$ et $\mathbf{B}[8]$.

2.1.2 Matériaux utilisés. - Nous nous proposons de déterminer expérimentalement les types de matériaux stables en compression dynamique et possédant néanmoins des caractéristiques piézoélectriques élevées.

Les matériaux sont choisis parmi les compositions peu lacunaires ou mểme stœchiométriques, proposées par L. Eyraud et al. [7, 8] et fabriquées par voie liquide. Leur formule générale est de la forme

$$
\mathrm{Pb}_{1-2 y} \mathrm{Sr}_{0,015} \mathrm{Ba}_{0,005} \mathrm{Na}_{y} \mathrm{~K}_{y}\left(\mathrm{Zr}_{x} \mathrm{Ti}_{1-x} \mathrm{Nb}_{0,02}\right) \mathrm{O}_{3,06} \text {. }
$$

Dans toute la suite, ils seront désignés par

$$
\text { VL } y \cdot x / 1-x \text {. }
$$

Pour chaque composition avec $y$ variable, la valeur de $x$ correspond au maximum du coefficient de couplage électromécanique. Pour la série VL 0,25 , les tests ont porté sur des compositions en $x$ situées de part et d'autre de ce maximum.

Deux compositions de type "doux" (VL Sb) et «dur» (VL Mn 52/48) ont été rajoutées à titre de comparaison; leurs compositions formulaires s'écrivent :

$$
\begin{aligned}
& \text { VL Sb : } \mathrm{Pb}_{0,85} \mathrm{Sr}_{0,12} \mathrm{Ba}_{0,05}\left(\mathrm{Zr}_{0,565} \mathrm{Ti}_{0,435} \mathrm{Sb}_{0,02}\right) \mathrm{O}_{3,07} \\
& \text { VL Mn : } \mathrm{PbZr}_{0,52} \mathrm{Ti}_{0,48} \mathrm{O}_{3}+0,008 \mathrm{MnO}
\end{aligned}
$$

2.1.3 Caractéristiques. - Le tableau I donne les principales caractéristiques piézoélectriques et électromécaniques de ces matériaux ainsi que l'énergie maximum $W_{\mathrm{M}}$ fournie sur charge capacitive lors d'un impact dans le percuteur à bille.

2.2 Stabilité SOUS ÉTINCELleS " IMPACt $20 \mu \mathrm{s}$ ". Le tableau II présente les variations relatives du coefficient $d_{33}$ après 400 impacts. Dans l'ensemble, les matériaux peu lacunaires présentent une très bonne stabilité, la variation d'une unité mesurée au piézomètre numérique Berlincourt-Channel n'étant pas significative. On note cependant un accroissement plus important du coefficient piézoélectrique pour les matériaux plus riches en $\mathrm{Zr}$ à $y$ constant. Seul le matériau " doux » VL Sb présente une perte sensible sur la valeur de $d_{33}$.

2.3 Stabilité en COURT-CIRCUIT. - Le comportement des céramiques sous compression rapide dépend de la valeur maximale de la contrainte appliquée mais également de la largeur de l'impulsion d'impact [5].

\begin{tabular}{|c|c|c|c|c|c|c|c|c|c|c|}
\hline \multirow{2}{*}{\multicolumn{2}{|c|}{ Type }} & VL 0 & VL 0,25 & VL 0,5 & VL 1 & \multicolumn{3}{|c|}{ VL 0,25} & \multirow{2}{*}{$\begin{array}{l}\mathrm{VL} \\
\mathrm{Sb}\end{array}$} & \multirow{2}{*}{$\begin{array}{c}\text { VL Mn } \\
52 / 48\end{array}$} \\
\hline & & $52 / 48$ & $53 / 47$ & $54 / 46$ & $54 / 46$ & $52 / 48$ & $53 / 47$ & $54 / 46$ & & \\
\hline$d_{33}$ & $\mathrm{pC} / \mathrm{N}$ & 414 & 510 & 485 & 432 & 418 & 510 & 348 & 800 & 225 \\
\hline$\varepsilon_{\mathrm{r}}$ & & 1885 & 1965 & 1975 & 1570 & 1994 & 1965 & 914 & $\underline{4310}$ & 1150 \\
\hline $\operatorname{tg} \delta$ & $\%$ & $\sim 2$ & $\sim 1$ & $\sim 1$ & $\sim 1$ & $\sim 1$ & $\sim 1$ & $\sim 1$ & $\sim 2,5$ & $\sim 0,5$ \\
\hline$g_{33}$ & $\mathrm{mV} \mathrm{m} / \mathrm{N}$ & 24,8 & 29,3 & 27,8 & 31,1 & 23,7 & 29,3 & $\underline{42}$ & 21 & 22,8 \\
\hline$F$ & $\mathrm{pm}^{2} / \mathrm{N}$ & 10,3 & 14,9 & 13,4 & 13,4 & 9,9 & 14,9 & 14,3 & $\underline{16,8}$ & 5,2 \\
\hline$k_{33}$ & & 0,778 & 0,775 & 0,750 & 0,720 & 0,729 & 0,775 & 0,757 & $\underline{0,783}$ & 0,64 \\
\hline$Q_{M}$ & & 83 & 59 & 54 & 55 & 61 & 59 & 51 & 35 & 476 \\
\hline$W_{\mathrm{M}}$ & $\mathrm{mJ}$ & 4,3 & 5,0 & 4,9 & 4,9 & 4,1 & 5,0 & 6,3 & 4,7 & 2,8 \\
\hline
\end{tabular}

2.3.1 Stabilité du coefficient piézoélectrique. - Les figures 7 et 8 présentent les variations relatives du

Tableau I. - Caractéristiques piézoélectriques et électromécaniques. 
Tableau II. - Variation du coefficient piézoélectrique sous impact rapide « $20 \mu \mathrm{s}$ » et sous étincelles.

\begin{tabular}{|c|c|c|c|c|c|c|c|c|c|}
\hline Type & VL 0 & VL 0,25 & VL 0,5 & VL 1 & \multicolumn{3}{|c|}{ VL 0,25 } & VL & VL Mn \\
& $52 / 48$ & $53 / 47$ & $54 / 46$ & $54 / 46$ & $52 / 48$ & $53 / 47$ & $54 / 46$ & Sb & $52 / 48$ \\
\hline$d_{330}$ & 414 & 510 & 485 & 432 & 418 & 510 & 348 & 756 & 224 \\
\hline$d_{33_{\mathbf{L}}}$ & 415 & 513 & 486 & 433 & 415 & 513 & 360 & 737 & 222 \\
\hline$\Delta d / d \% /$ & $\sim+2$ & +6 & $\sim+2$ & $\sim+2$ & -7 & +6 & +34 & -25 & -9 \\
\hline
\end{tabular}

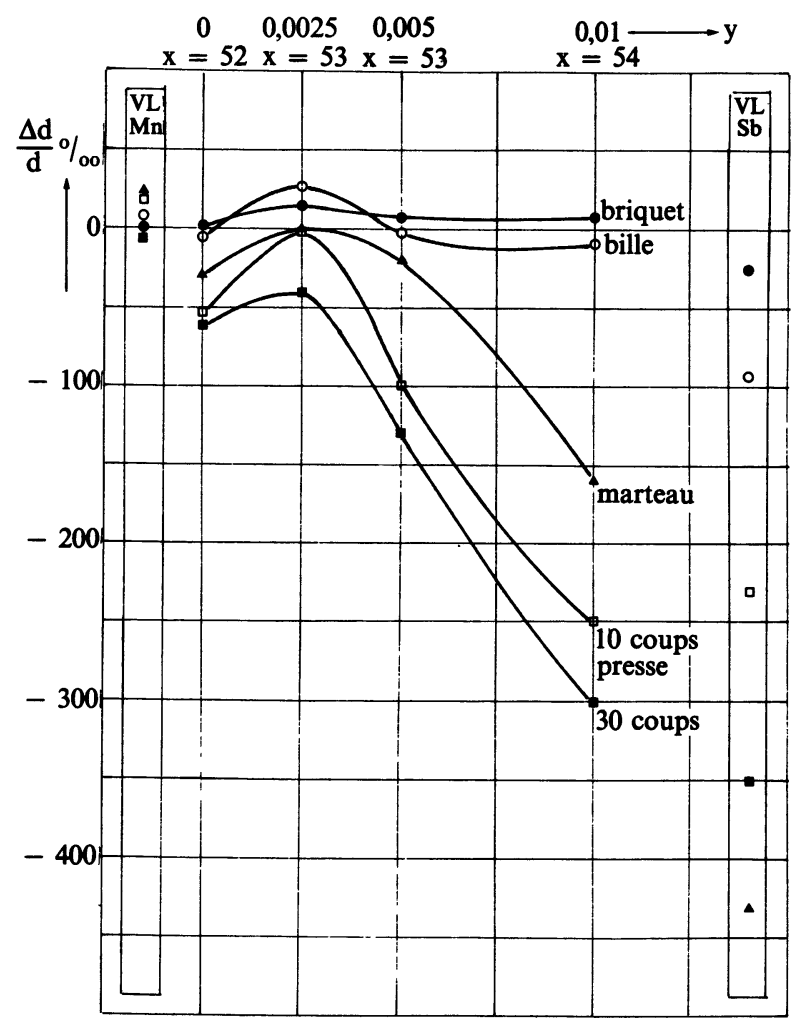

Fig. 7. - Variation relative du coefficient piézoélectrique en fonction de $y=\% \mathrm{Na}, \mathrm{K}$.

[Dependence of relative piezoelectric coefficient on the compositional variable $y$.]

coefficient piézoélectrique des matériaux, obtenues lors des cinq essais : briquet, bille, marteau, compression lente 600 bars, 10 coups puis 30 coups.

D'une manière générale, l'élargissement de la durée de l'impact accélère la détérioration du coefficient $d_{33}$. A l'exception du matériau "doux", toutes les compositions sont stables à " $20 \mu \mathrm{s}$ » et presque toutes à " $100 \mu \mathrm{s}$ ». Si la céramique de "squeeze" VL Mn 52/48 est effectivement stable même à 30 montées en compression lente, on note les excellentes performances de la céramique VL $0,25.53 / 47$; cette composition à grand coefficient de couplage électromécanique $\left(k_{33}=0,775\right)$ présente l'avantage de délivrer une très forte énergie sur capacité $\left(W_{\mathrm{M}} \simeq 5 \mathrm{~mJ}\right.$. La figure 8 montre qu'une modification de la teneur

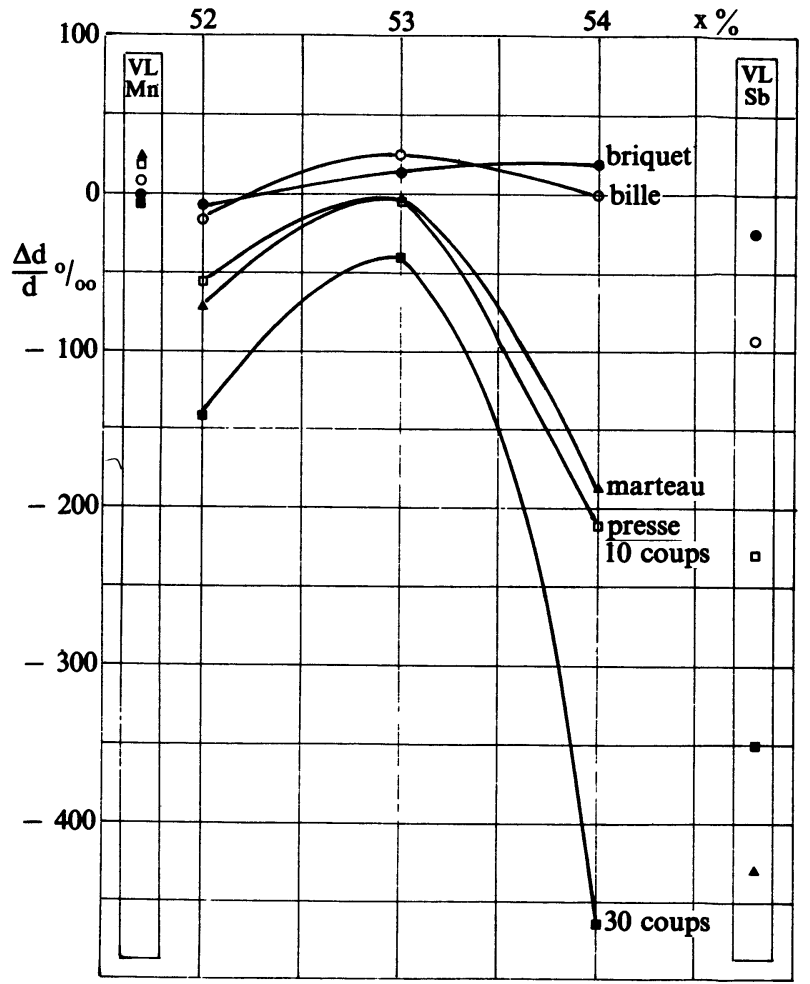

Fig. 8. - Variation relative du coefficient piézoélectrique en fonction de $x=\% \mathrm{Zr}$.

[Dependence of relative piezoelectric coefficient on the compositional variable $x$.]

en zirconium de la composition VL 0,25 dégrade notablement sa stabilité.

2.3.2 Charges écoulées en court-circuit. - Les figures 9 et 10 comportent les courbes suivantes :

$a$ - maximum $Q_{\mathrm{m}}$ des charges écoulées en courtcircuit au cours des impacts au percuteur à bille (valeur obtenue après quelques chocs pour assurer le contact)

$b$ - variation relative $\Delta Q / Q$ après 400 essais au percuteur à bille,

$c$ - charge $Q_{1}$ libérée au cours de la première montée en compression lente à 600 bars,

$d$ - variation relative $\Delta Q / Q$ après 10 compressions lentes. 


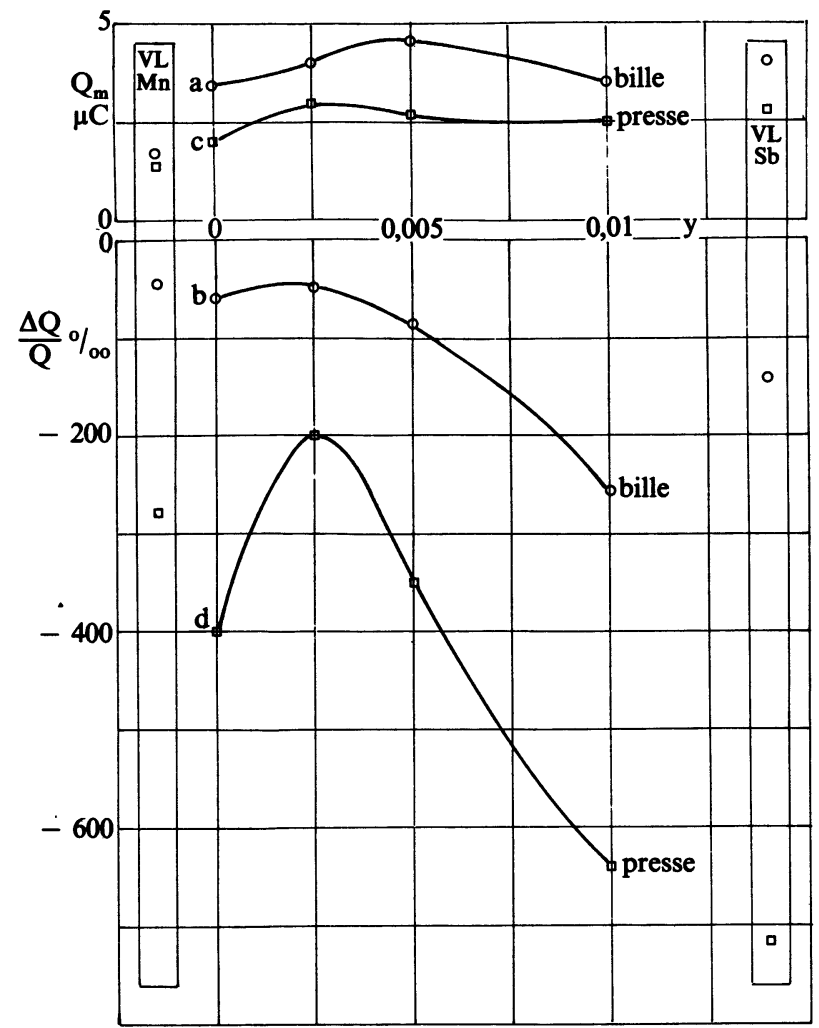

Fig. 9. - Charges écoulées en fonction de $y$.

[Released electrical charges versus $y$.]

Ces mesures confirment la très bonne stabilité de la composition VL 0,25.53/47.

On remarque que si les charges étaient libérées uniquement sous l'influence d'un effet piézoélectrique linéaire réversible et indépendant du temps de montée de la compression, on devrait observer $Q_{\mathrm{m}}=2 Q_{1}$ $\left(Q_{\mathrm{m}}\right.$ correspond à la somme des charges libérées à la montée et à la descente). En fait, à l'exception de la céramique VL $0,25.52 / 48$, on a $Q_{\mathrm{m}}<2 Q_{1}$ ce qui indique une libération plus importante de charges en compression quasi statique. Toutefois la réversibilité est plus mauvaise puisque la perte $\Delta Q / Q$ est déjà très importante au bout de 10 montées.

Par ailleurs, la comparaison des courbes $\Delta d / d$ et $\Delta Q / Q$ montre que la diminution des charges écoulées est plus importante que la baisse du coefficient $d_{33}$. Ceci traduit une linéarisation de la caractéristique $Q=f(T)$ au cours des impacts successifs.

\subsubsection{Charges libérées lors d'une compression à} 3000 bars. - La dépolarisation quasi totale de la céramique est mise en évidence par la faible valeur du coefficient piézoélectrique mesuré après l'expérience. On note sur la figure 11 que pour les composés à $y$ constant et $x$ variable, la pente est d'autant plus faible que le pourcentage en $\mathrm{Zr}$ est moins élevé (courbes e, c, f); en revanche la courbe e présente une plus grande linéarité ce qui correspond bien à la céramique pour laquelle $Q_{\mathrm{m}} \simeq 2 Q_{1}$.

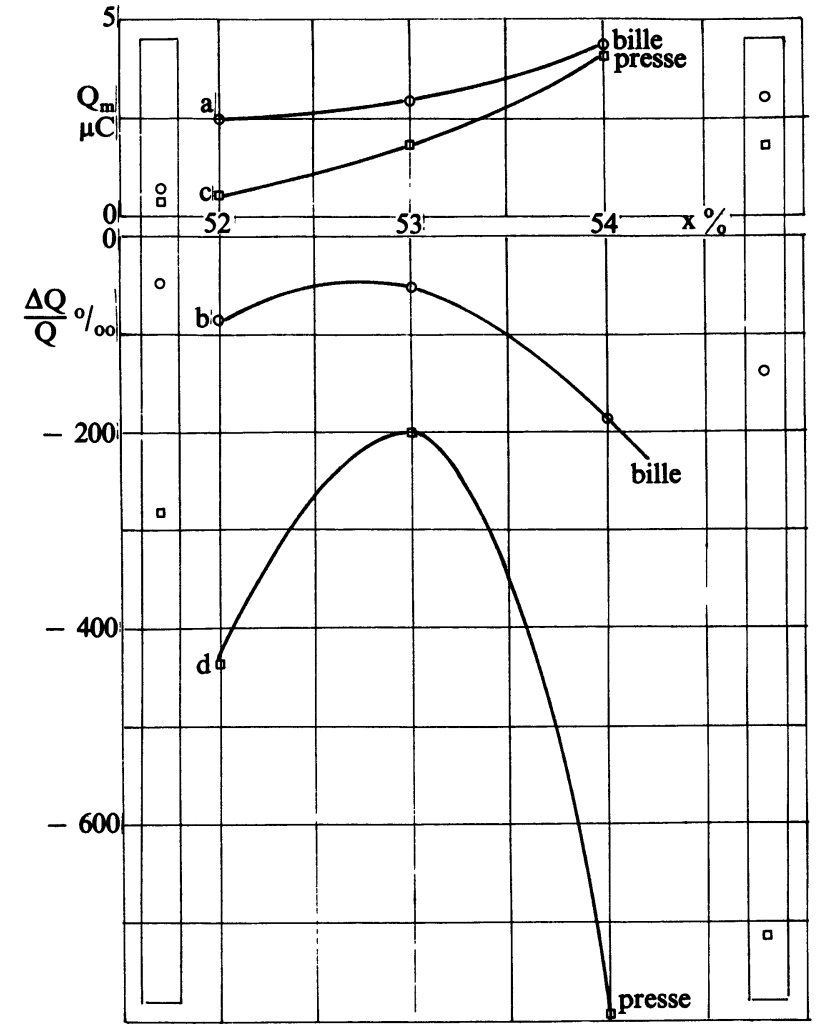

Fig. 10. - Charges écoulées en fonction de $x$.

[Released electrical charges versus $x$.]

D'autre part, l'augmentation du pourcentage en $\mathrm{Na}$, $\mathrm{K}$ produit le même effet, quoique atténué, qu'une diminution de la teneur en zirconium (courbes $a, b, f$ ).

2.3.4 Analyse du mélange de phases. - Le tableau III indique les pourcentages de phase rhomboédrique (R) déterminés par diffraction $X$ avec la méthode de dépouillement décrite au $\S 1.3$. Comme prévu, ce pourcentage augmente avec le pourcentage en zirconium [10]. L'augmentation du pourcentage en $\mathrm{Na}, \mathrm{K}$ a tendance à diminuer le pourcentage de phase rhomboédrique. Cependant, les propriétés piézoélectriques optimales correspondent toujours à environ $30 \%$ de phase $R$. Ces résultats corroborent ceux observés lors de la dépolarisation quasi statique à 3 kbars (Fig. 11).

\section{Conclusion.}

Cette étude fait apparaître que le choix d'un matériau piézoélectrique à la fois performant et stable en compression dynamique est assez critique.

En premier lieu, pour une amplitude donnée de l'impulsion de pression, la stabilité du matériau est une fonction décroissante de sa durée et donc du mécanisme d'utilisation. Deux éléments de la composition formulaire du matériau ont une influence prépondérante : d'une part, le rapport entre la concentration 


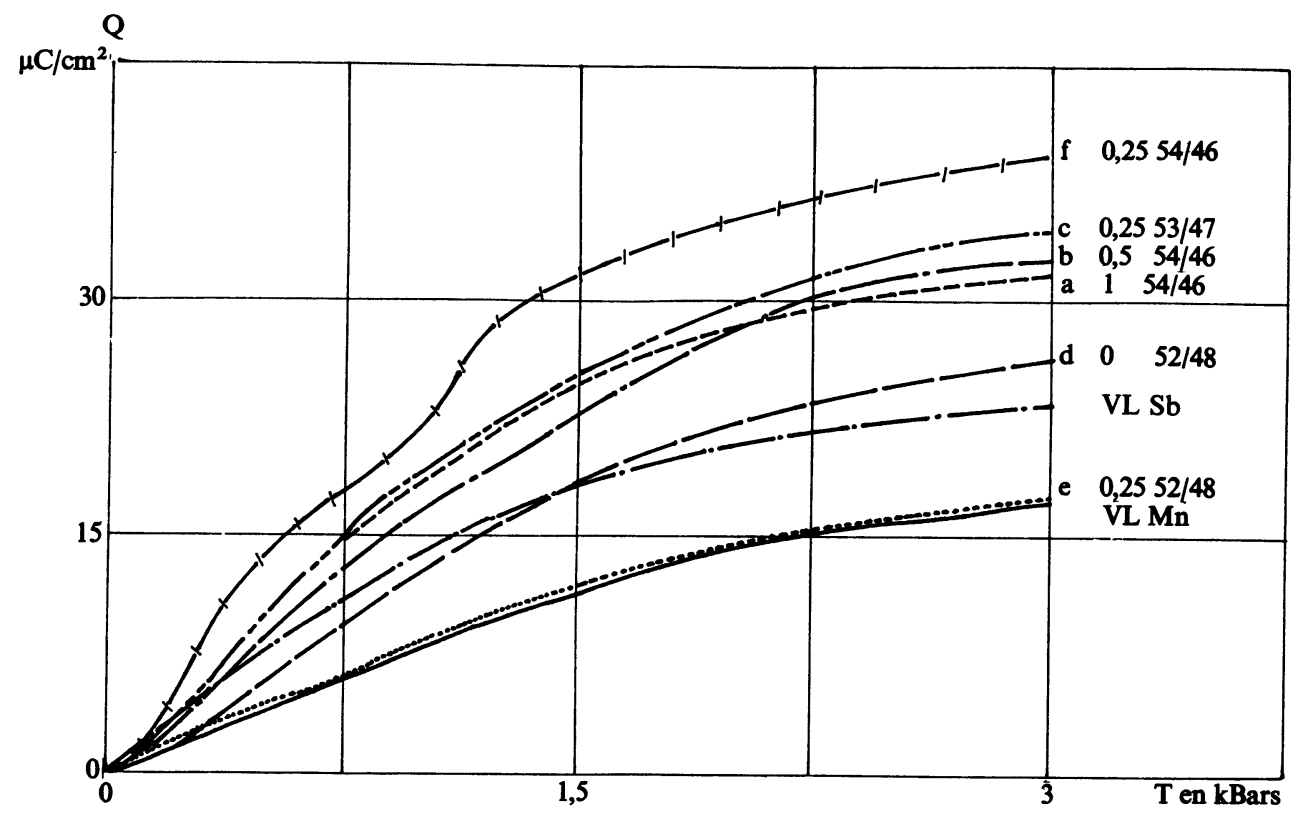

Fig. 11. - Charges écoulées en fonction de la pression.

[Released electrical charges as a function of pressure.]

Tableau III. - Pourcentage de phase rhomboédrique dans le matériau.

\begin{tabular}{|c|c|c|c|c|c|c|c|c|c|}
\hline Type & VL 0 & VL 0,25 & VL 0,5 & VL 1 & \multicolumn{3}{|c|}{ VL 0,25 } & VL & VL Mn \\
& $52 / 48$ & $53 / 47$ & $54 / 46$ & $54 / 46$ & $52 / 48$ & $53 / 47$ & $54 / 46$ & Sb & $52 / 48$ \\
\hline$\% \mathbf{R}$ & 16 & 30 & 28 & 27 & 10 & 30 & 65 & 27 & 19 \\
\hline
\end{tabular}

en sodium/potassium et la concentration en niobium permet de modifier l'état lacunaire et l'équilibre électrique [8] de la composition; d'autre part la concentration en zirconium/titane doit impérativement correspondre au maximum du coefficient de couplage électromécanique; ce maximum est obtenu pour environ un tiers de phase rhomboédrique. La seule composition délivrant une forte énergie et qui résiste aux trois tests est VL $0,25.53 / 47$ qui comporte $x=53 \%$ de zirconium et $y=0,25 \%$ de sodium et potassium. Des essais effectués sur des matériaux élaborés par voie solide montrent un comportement analogue aux précédents mais avec une fiabilité beaucoup moins bonne.

\section{Bibliographie}

[1] Ohanessian, H., Gonnard, P., Rev. Phys. Appl. 16 (1981) 647-655.

[2] Ohanessian, H., Gonnard, P., Fétiveau, Y., Rev. Phys. Appl. 9 (1974) 995-999.

[3] Mabud, S. A., J. Appl. Cryst. 13 (1980) 211-216.

[4] Thomann, H. Z., Angew Phys. 20 (1966) 554.

[5] Banno, H., Tsunooka, T., Elect. Ceram. 3, 10 (1972) 36-40.
[6] Takahashi, S., Ferroelec. 27 (1980) 109-112.

[7] Eyraud, L., Eyraud, P., Gonnard, P., Troccaz, M., Ferroelec. 34 (1981) 133-138.

[8] Eyraud, L., Eyraud, P., Mathieu, J. C., C.R. Hebd. Séan. Acad. Sci. (1982).

[9] Notice Technique Quartz \& Silice 1974.

[10] Wersing, H., Ferroelec. 7 (1974) 163-165. 